Zukunftsfähige Produktions- und Konsummuster

\title{
Megatrends und die grüne Wirtschaftspolitik
}

\begin{abstract}
Um Wirtschaftssysteme in Bezug auf ihre Umwelteffekte nachhaltiger zu gestalten, bedarf es nicht nur politischer Leitbilder. Vor allem die umfassende Berücksichtigung sozialer und technologischer Entwicklungen im Kontext von Produktion und Konsum ist entscheidend. Von Jan-Peter Ferdinand
\end{abstract}

D ie Zukunftsfähigkeit von Wirtschaftssystemen entscheidet sich in den nächsten Jahrzehnten vor allem durch ihre Einbettung in sozio-ökologische Kontexte. So beschreiben aktuelle Konzepte einer "Low Carbon Economy" oder des „Green Growth“ mögliche Pfade einer sogenannten grünen Ökonomie, die Kriterien einer nachhaltigen Entwicklung mit Bedingungen wirtschaftlichen Wachstums zu verknüpfen versuchen (Europäische Kommission 2011; OECD 2011).

\section{Nachhaltige Wachstums- strategien}

Indem diese Ansätze vor allem der programmatischen Orientierung dienen, richten sich entsprechende Diskussionsprozesse zunächst primär auf Optionen grüner Wachstumsstrategien. Diese Strategien haben im Wesentlichen zum Ziel, die Handlungsweisen von Unternehmen und Konsumenten in nachhaltigere Bahnen zu lenken und Investitionen in neue Technologien und Prozesse als grüne Innovationen anzustoßen.

Obwohl solche grundlegenden Orientierungen einen sinnvollen Schritt in Richtung nachhaltiger Wirtschaftsformen darstellen, bedarf es auch einer baldigen Fundierung dieser Konzepte hinsichtlich konkreter ökonomischer Zusammenhänge. Erste Ansätze setzte hierzu vor Kurzem das United Nations Environment Programme (UNEP) um, das in der Auseinandersetzung mit der grünen Ökonomie eine erste Verknüpfung von Po- tenzialen für die Energie- und Ressourceneffizienz mit Gestaltungsmöglichkeiten von Wirtschaftssystemen vorschlägt (UNEP 2011).

Eine weiterführende sozial-ökologische Analyse dieser Zusammenhänge müsste explizit Fragestellungen grundsätzlicher Visionen und Optionen für nachhaltigere Formen des Wirtschaftens in den Blick nehmen und diese mit handlungsleitenden Entwicklungsdynamiken in Bezug setzen. Am Beispiel des Themenfeldes der Produktions- und Konsummuster, das sowohl Formen der Wertschöpfung als auch den Bereich konsumseitiger Nachfrage berücksichtigt, soll exemplarisch gezeigt werden, welche prinzipiellen Anknüpfungspunkte zwischen den nachhaltigkeitsbezogenen Leitbildern und konkreteren technologischen beziehungsweise sozialen Innovationsfeldern existieren.

\section{Von grüner Wirtschafts- politik ...}

In inhaltlicher Nähe zum skizzierten politischen Diskurs stehen im Kontext zukunftsfähiger Produktionsstrukturen zumindest im europäischen Raum zwei Ansätze im Fokus, die Nachhaltigkeitsaspekte in unterschiedlicher Deutlichkeit adressieren.

Einerseits geht es um Übergänge hin $z u$ einer wertsteigernden und wissensbasierten Produktionsstrategie, die verstärkt auf kundenspezifische und/oder hochleistungsfähige Produkte setzt, de- ren Herstellung extrem wissenssensibel und serviceintensiv ist. Digital Manufacturing gilt in diesem Zusammenhang als technologisches Leitbild für intelligente Produktionssysteme, die sich adaptiv und flexibel auf sich verändernde Rahmenbedingungen einstellen können (Monostori et al. 2010).

Anderseits verweisen Zugänge wie die europäische Factories of the future-Roadmap (2010) explizit auf die Notwendigkeit nachhaltiger Produktionsmuster. In diesem Zusammenhang sind auch Forschungsaktivitäten zu sehen, die Themen eines hocheffizienten, nahezu emissionslosen Fertigungsprozesses betreffen, Alternativen zu energieintensiven Herstellungsverfahren suchen oder die Einsatzmöglichkeiten erneuerbarer, klimaneutraler Rohstoffe als industrielles Grundmaterial ermitteln wollen.

\section{... zu grünen Produktions- und Konsummustern}

Nachhaltigkeit bildet das gemeinsame Ziel dieser zunächst technologisch orientierten Entwicklungspfade, wobei neben ökologischen auch ökonomische und soziale Aspekte angesprochen werden. Eine Schwäche der bisherigen Auseinandersetzung liegt neben der ausbaufähigen Integration der verschiedenen politischen Handlungsfelder auch in der relativ eingeschränkten Deutung der berücksichtigten Zusammenhänge.

So haben sich zum Beispiel auf der Ebene des alltäglichen Lebens einige soziale Innovationen herausgebildet, die Produktions- und Konsummuster zukünftig beeinflussen können. Obwohl in diesem Kontext Konzepte wie „Nutzen statt Besitzen“, „Cradle to Cradle“ oder „FabLabs" aktuell in unterschiedlichsten Anwendungsfeldern an Relevanz gewinnen, finden sie in der innovationspolitischen Auseinandersetzung um zukunftsfähige Produktions- und Konsummuster nur selten Beachtung.

Im Rahmen eines Forschungsvorhabens lotet das IÖW derzeit aus, wie aktuelle Megatrends wie Globalisierung, Individualisierung oder Klimawandel vor dem Hintergrund der Leitbilder einer 
grünen Wirtschaft mit zukunftsrelevanten Innovationen im Bereich von Produktion und Konsum interagieren. Im Rahmen des Projekts wird geprüft, welche Effekte Gestaltungsoptionen wie Dezentralisierung oder Nutzerintegration auf die Zukunftsfähigkeit von Wirtschaftssystemen haben und wie Konzepte von „resilient regions“ oder „community production“ in diesem Zusammenhang $\mathrm{zu}$ bewerten sind.

\section{Ausblick}

Obwohl die Leitbilder einer grünen Wirtschaft prinzipiell vielfältige Anknüpfungspunkte sowohl zu gesellschaftlichen Megatrends als auch zu diversen Innovationsbereichen aufweisen, wurden diese bisher noch nicht systematisch in die Konzeption einer Gesamtstrategie einbezogen. Auch wird im Rahmen erster förderpolitischer Aktivitäten die Bedeutung sozialer Innovationen weitgehend vernachlässigt. Deren Berücksichtigung ist jedoch gerade im Kontext zukunftsfähiger Produktions- und Konsummuster notwendig, da der Einfluss potenzieller Kunden auf den Produktionsprozess, sei es über die Reaktion auf Nachfragesituation oder die unmittelbare Integration von Nutzern in Produktentstehungsprozesse, einen wesentlichen Trend für Wirtschaftssysteme darstellen wird. Insofern werden es sowohl technologische als auch lebensweltliche Aspekte sein, die die Zukunftsfähigkeit von Wirtschaftssystemen im Kern beeinflussen.

Perspektivisch wird es darauf ankommen, die Konzeption von Umweltverträglichkeit und Nachhaltigkeit stärker in wirtschaftsrelevante Diskurse zu integrieren und die Gestaltung sinnvoller technologischer wie sozialer Innovationen in entsprechende Richtungen zu leiten.

\section{Literatur}

Ad-hoc Industrial Advisory Group of the Factories of the Future PPP: Factories of the Future PPP Strategic Multi-annual Roadmap. 2010. Internet: http://www.manufuture.org/manufacturing/wp-content/uploads/FoF_PPP_ Roadmap_Final_Version.pdf

Europäische Kommission: A Roadmap for moving to a competitive low carbon economy in 2050 .
2011. Internet: http://eur-lex.europa.eu/ LexUriServ/LexUriServ.do?uri=COM:2011: 0112:FIN:EN:PDF

Monostori, L. et al.: Towards adaptive and digital manufacturing. In: Annual reviews in Control 34, $1 / 2010$, S.118-128.

OECD: Towards Green Growth. 2011. Internet: http://www.oecd.org/dataoecd/37/34/48224539.pdf

UNEP: Towards a Green Economy: Pathways to Sustainable Development and Poverty Eradication. 2011. Internet: http://www.unep.org/greeneconomy/ Portals/88/documents/ger/GER_synthesis_ en.pdf

\section{【 AUTOR+ KONTAKT}

Jan-Peter Ferdinand ist wissenschaftlicher Mitarbeiter im Forschungsfeld Umweltökonomie und -politik am Institut für ökologische Wirtschaftsforschung.

Jan-Peter Ferdinand,

Institut für ökologische Wirtschaftsforschung, Potsdamer Straße 105, 10785 Berlin. Tel.: +49 $30884594-28$ E-Mail: jan-peter.ferdinand@ioew.de

\section{Sprit aus der Steckdose}

Unsere Metropolen brauchen neue Verkehrskonzepte, unsere Fahrzeuge neue Antriebe. Klimawandel, Rohstoffknappheit und der drohende Verkehrsinfarkt erzwingen einen Wechsel hin zu postfossilen Energieträgern. Die Lösung heißt: vernetzte Elektromobilität. Sie kann die Bewegungsfreiheit schaffen, die moderne Gesellschaften benötigen. Weert Canzler und Andreas Knie skizzieren den Einstieg in eine neue Mobilität, die eine Verknüpfung von öffentlichen und privaten Verkehrsmitteln erreicht - und das vollständig auf Basis erneuerbarer Energien.

W. Canzler, A. Knie Einfach aufladen Mit Elektromobilität in eine saubere Zukunft

124 Seiten, Klappenbroschur, 9,95 EUR, ISBN 978-3-86581-270-4

\section{/ll oekom}

Die guten Seiten der Zukunft

Erhältlich bei www.oekom.de oekom@verlegerdienst.de

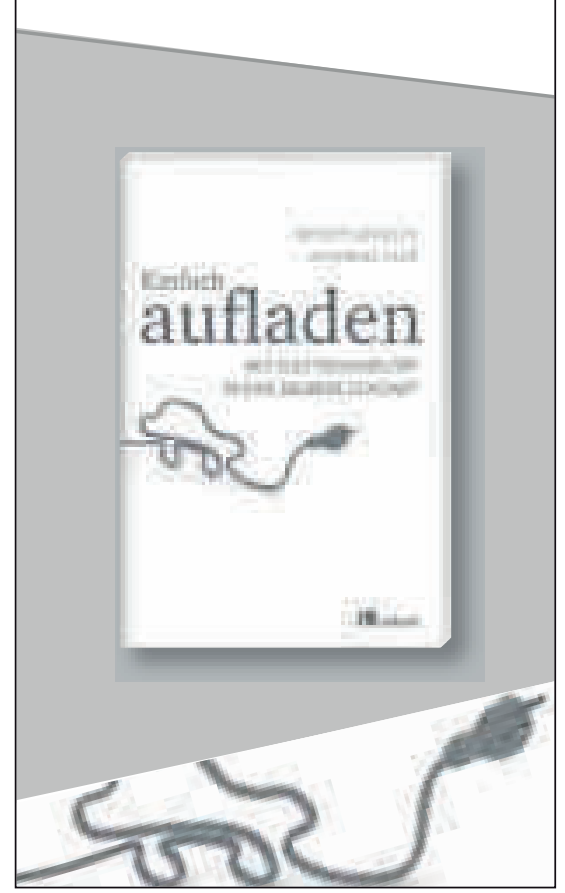




\section{Lizenzhinweis}

Die Beiträge in ÖkologischesWirtschaften werden unter der Creative-Commons-Lizenz "CC 4.0 Attribution Non-Commercial No Derivatives" veröffentlicht. Im Rahmen dieser Lizenz muss der Autor/Urheber stets genannt werden, das Werk darf nicht bearbeitet, abgewandelt oder in anderer Weise verändert und außerdem nicht kommerziell genutzt werden. Die digitale Version des Artikels bleibt für zwei Jahre Abonnent/innen vorbehalten und ist danach im Open Access verfügbar. 